\title{
Fatal Drug Rash
}

Sami L. Bahna *, Diana Munoz-Mendoza, Neetu Godhwani

From the Allergy/Immunology Section, Louisiana State University Health Sciences Center, Shreveport, Louisiana, USA

${ }^{*}$ Corresponding Author: Sami L. Bahna, From the Allergy/Immunology Section, Louisiana State University Health Sciences Center, Shreveport, Louisiana, USA. E-mail:SBahna@Isuhsc.edu

Received date : July 11, 2019; Accepted date : July 26, 2019; Published date : July 30, 2019

Citation : Sami L. Bahna (2019) Fatal Drug Rash.J Dermatol Dermatit 4(2) DOI: 10.31579/2578-8949/052

Copyright : (c) 2019 Sami L. Bahna. This is an open-access article distributed under the terms of The Creative Commons Attribution License, which permits unrestricted use, distribution, and reproduction in any medium, provided the original author and source are credited.

\section{Abstract}

We describe the case of a woman presenting with rash and fever 3 weeks after starting oxcarbazepine. She had a convoluted, prolonged hospital course involving reactions to multiple structurally-different medications, with a variety of severe manifestations including drug rash with eosinophilia and systemic symptoms (DRESS), and Stevens-Johnson syndrome/toxic epidermal necrolysis (SJS/TEN), and complicated with MRSA septic shock and death. This case illustrates the high risk in subjects with a drug reaction that initiated excessive $\mathrm{T}$ cell stimulation with subsequent reactions to multiple drugs.

Keywords: Drug allergy;drug rash with eosinophilia and systemic symptoms (DRESS); Stevens-Johnson syndrome (SJS); toxic epidermal necrolysis (TEN);multiple drug hypersensitivity syndrome

\section{Case Report}

Some rashes constitute a diagnostic and therapeutic dilemma as in the case presented here.

A 38-year-old female was admitted for a generalized rash and fever. Eight days earlier, she developed fever $\left(103^{\circ} \mathrm{F}\right)$, shortness of breath, and a rash starting on her back and face and became generalized and associated with facial swelling, without improvement on diphenhydramine. One month prior to admission, she was taking oxcarbazepine for bipolar disorder, and it was discontinued when the rash appeared. Her past medical history and family history were noncontributory.On admission, she had T $99.5^{\circ} \mathrm{F}$, HR 145/min, RR 20/min, and BP 105/72 $\mathrm{mm} \mathrm{Hg}$.
Physical findings included scleral icterus, posterior cervical lymphadenopathy of $2 \mathrm{~cm}$, enlarged liver $1.5 \mathrm{~cm}$ below the right costal margin, and a diffuse confluent, erythematous rash with some pustular lesions on her arms, and lower extremities, as well as mild exfoliation on the neck. There was no mucosal involvement.

Laboratory evaluation (Table I, Figure 1) showed leukocytosis $(20,280 / \mu \mathrm{L})$, eosinophilia $(910 / \mu \mathrm{L})$, liver transaminitis (AST $82 \mathrm{U} / \mathrm{L}$, ALT $211 \mathrm{U} / \mathrm{L}$, ALP $305 \mathrm{U} / \mathrm{L})$, elevated bilirubin $(2.8 \mathrm{mg} / \mathrm{dL})$, and elevated procalcitonin $(3.76 \mathrm{ng} / \mathrm{mL}$; normal $<0.5)$. Serum lactate was slightly elevated (1.6 mmol/L; normal 0.4-2.0). Serum acetaminophen was not at a toxic level and she had negative HIV and hepatitis panels.

\begin{tabular}{|c|c|c|c|c|c|c|c|c|}
\hline Hospital day & WBC $($ cells $/ \mu \mathrm{L})$ & Eos $($ cells $/ \mu \mathrm{L})$ & $\operatorname{AST}(\mathrm{U} / \mathrm{L})$ & ALT (U/L) & $\operatorname{ALP}(\mathrm{U} / \mathrm{L})$ & Total bili (mg/dL) & INR & HHV-6 (DNA copies/mL) \\
\hline Normal & $3.6-11.2$ & $0-0.5$ & $15-37$ & $12-78$ & $45-117$ & $0.2-1.0$ & $<1.1$ & $<188$ \\
\hline 1 & 20.28 & 910 & 82 & 211 & 305 & 2.8 & 1.62 & \\
\hline 2 & 16.53 & 496 & 95 & 186 & 240 & 2.5 & 1.53 & \\
\hline 3 & 17.69 & 1415 & 174 & 209 & 214 & 2.9 & & \\
\hline 4 & 21.39 & 1925 & 260 & 306 & 215 & 3.5 & 1.81 & \\
\hline 5 & 26.42 & 4227 & 374 & 492 & 249 & 3.2 & & \\
\hline 6 & 23.35 & 3269 & 630 & 763 & 273 & 3.4 & 1.58 & $<188$ \\
\hline 7 & 20.65 & 2065 & 750 & 791 & 300 & 4.4 & 1.49 & \\
\hline 8 & 22.91 & 3460 & 1037 & 893 & 402 & 7.7 & 1.98 & \\
\hline 9 & 17.55 & 527 & 928 & 1084 & 346 & 9.9 & 2.08 & \\
\hline 10 & 20.96 & 419 & 430 & 936 & 338 & 11 & 1.7 & \\
\hline 11 & 22.91 & 345 & 386 & 883 & 358 & 13.4 & 1.51 & \\
\hline 12 & 17.55 & 133 & 474 & 909 & 337 & 13.6 & 1.49 & \\
\hline 13 & 20.96 & 0 & 911 & 1389 & 329 & 16.1 & 1.54 & \\
\hline 14 & 16.39 & 0 & 930 & 1672 & 304 & 16.6 & 1.53 & \\
\hline 15 & 13.6 & 0 & 493 & 1432 & 285 & 15.4 & 1.51 & 375 \\
\hline 23 & 11.23 & 0 & 67 & 170 & 140 & 5 & & \\
\hline 24 & 10.8 & 0 & 90 & 193 & 176 & 4.1 & & \\
\hline 25 & 11.87 & 0 & 99 & 216 & 190 & 3.7 & & \\
\hline 54 & 5.08 & 0 & 156 & 285 & 388 & 5.9 & & \\
\hline 56 & 5.99 & 0 & 146 & 249 & 362 & 4.6 & & \\
\hline 60 & 6.18 & 0 & 628 & 699 & 613 & 7.4 & & \\
\hline 70 & 4.26 & 0 & 91 & 145 & 378 & 10.4 & & \\
\hline
\end{tabular}

Table 1: Select laboratory findings during hospitalization of a woman with multiple drug hypersensitivity syndrome. 
$\mathrm{ALP}=$ alkaline phosphatase $;$ ALT $=$ alanine aminotransferase $;$ AST = aspartate aminotransferase; Bili= bilirubin; Eos = eosinophils; HHV-6 $=$ Human Herpesvirus 6, INR = International normalized ratio.

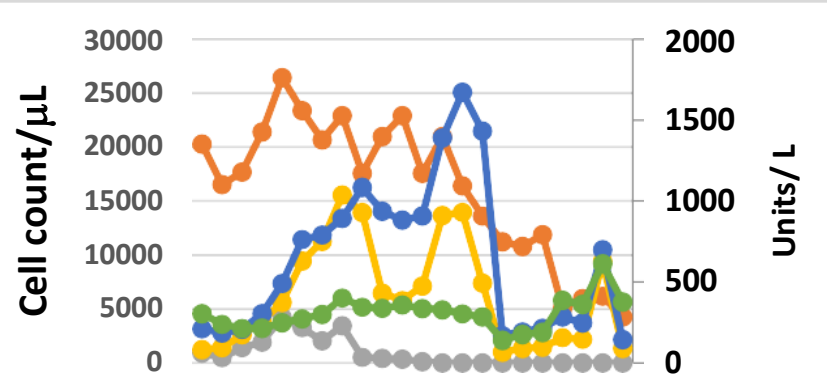

13579111315245460

Hospitalization day

$\because \mathrm{WBC} \rightleftharpoons \mathrm{EOS} \backsim \mathrm{AST} \longrightarrow \mathrm{ALT} \multimap \mathrm{ALP}$

Figure 1. Course of laboratory findings during hospitalization of a woman with multiple drug hypersensitivity syndrome.

Our initial differential diagnoses included: drug rash with eosinophilia and systemic symptoms (DRESS), drug-induced hepatitis, acute generalized exanthematous pustulosis (AGEP), Stevens-Johnson syndrome/toxic epidermal necrolysis (SJS/TEN), and sepsis.

Because of concern about infection, vancomycin and piperacillintazobactam were administered but discontinued when the blood and urine cultures showed no growth. Drug-induced hepatitis to oxcarbazepine was considered, either alone or as a part of DRESS. AGEP was unlikely because of the hepatic involvement, and systemic symptoms. It usually presents as diffuse non-follicular sterile pustules on erythematous bases [1]. SJS/TEN seemed unlikely because of the appearance of the rash and lack of mucositis.

Methylprednisolone $1.5 \mathrm{mg} / \mathrm{kg} / \mathrm{day}$ was initiated. She remained febrile and tachypneic, and by day 7 the rash spread to her palms and legs, with exfoliation on her back and arms. To enhance elimination of oxcarbazepine metabolites, n-acetylcysteine was administered but was discontinued within 10 minutes of infusion because of facial angioedema and chills.

Serum human herpesvirus-6 PCR was elevated as well as increased transaminases (AST 474U/L, ALT 909 U/L, ALP 329 U/L) and bilirubin (16 mg/dL).

A week later, the patient developed painful, pearly nodular lesions on abdomen and axilla then became febrile $\left(101.7{ }^{\circ} \mathrm{F}\right)$, hypotensive $(90 / 50 \mathrm{~mm} \mathrm{Hg})$, and tachycardic (169/min). Blood cultures and skin biopsy showed gram-positive cocci in clusters and she was started on IV daptomycin. The next day, she had severe chest pain and ultrasound showed left chest wall abscess and pericardial tamponade from which $400 \mathrm{~mL}$ of purulent fluid was aspirated. Two days later, daptomycin was substituted with ceftaroline due to better toxicity profile, and methylprednisolone was tapered over 1 week. A pericardial window was performed, the abscess was evacuated, and a drain was placed. Methicillin-resistant staphylococcus aureus grew in cultures from the pericardial, pleural, and peritoneal effusions.

She subsequently had altered mental status, emesis and multiple episodes of seizures treated with topiramate. CSF had no leukocytes and culture was negative. While on topiramate for 2 weeks, erythematous macular rash appeared on her palms and soles. Punch biopsy was consistent with erythema multiforme. Topiramate was replaced with levetiracetam, but was discontinued 10 days later due to development of oral mucositis consistent with SJS. Liver biopsy revealed acute hepatitis with lymphocytic infiltrate suggestive of toxic reaction.

Ten weeks after initial hospitalization she developed rapidly evolving TEN that within 24 hours encompassed $90 \%$ of her body.

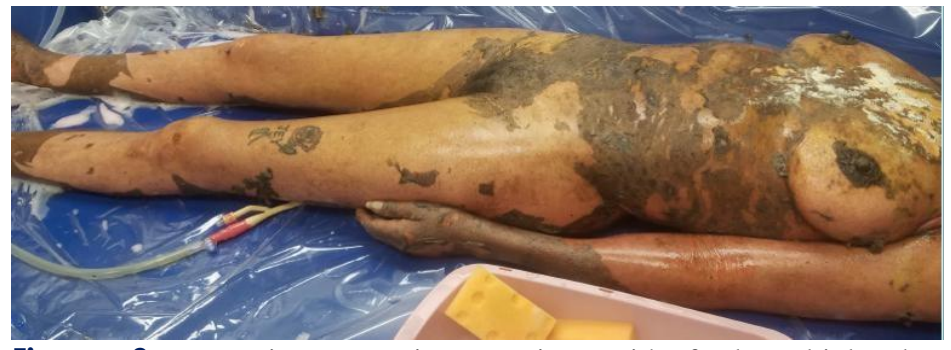

Figure 2: Extensive TEN in a patient with fatal multiple drug hypersensitivity syndrome.

Intravenous immunoglobulin (IVIG) $1 \mathrm{~g} / \mathrm{kg}$ was administered at $24-\mathrm{hr}$ intervals for 3 doses, with slight improvement. Ceftaroline and gentamycin were administered for presumed sepsis and the blood cultures grew pan-sensitive Serratia marcesens. HSV-PCR and mycoplasma-IgM antibody were negative. She developed pulmonary edema, hypovolemic shock, and acute kidney injury. Plasmapheresis was performed and repeated the next day, but she went into cardiac arrest and could not be resuscitated. Autopsy was not done.

\section{Discussion}

Several important points can be learned from this patient's convoluted, prolonged course that ended in death despite close monitoring and aggressive treatment. Our patient reacted to many drugs with rapid onset of different severe manifestations. Furthermore, the course was confounded by sepsis with MRSA and Serratia marcesnes complicated with purulent pericarditis, empyema, peritonitis, and cardiac tamponade.

The initial reaction was compatible with DRESS attributed to oxcarbazepine. Improvement started after initiating corticosteroid therapy. Administration of anticonvulsants might have suppressed the humoral immunity causing reactivation of HHV-6 which can worsen the prognosis of DRESS [2-4]. Within 24 hours of starting pantoprazole, the liver enzymes markedly increased -- more likely secondary to DRESS than to this medication [5]. Within minutes of infusing $\mathrm{N}$-acetylcysteine, she developed facial angioedema and chills, which occurs in $<1 \%$ of patients receiving this drug [6]. Topiramate seems to start the march of the SJS/TEN followed with reactions to multiple drugs. It seems that the sepsis was secondary to the extensive TEN. Consequently she developed chest abscess and pericarditis, with subsequent deteriorating fatal course.

Despite aggressive specialized Burn Unit care, IVIG therapy, and plasmapheresis, our patient did not recover. SJS/TEN can be associated with high fatality despite a high IVIG dose [7] or early administration [8].

Two studies on Chinese populations showed an association of HLAB*15:02 and the development of SJS/TEN to oxcarbazepine [9-10]. Could an underlying immune defect be a cause for the overwhelming sepsis that involved multiple vital organs? This adult patient had no past history of recurrent infections. Aromatic anti-epileptics, such as oxcarbazepine, which was taken for 3 weeks, can cause immunosuppression [11].

Our patient reacted to multiple structurally unrelated drugs with different manifestations, compatible with MDH [12]. An initial severe reaction is usually T-cell mediated, often DRESS, and the subsequent reactions generally have different mechanisms. In other words, the magnitude of the initial T-cell stimulation appears to be the driving factor for development of MDH. Verification of the offending agents after recovery can be a challenging task. Skin testing, basophil activation, and lymphocyte transformation testing might be useful, but their availability, sensitivity and specificity are not high [12-14]. One study found that up to a quarter of patients with DRESS are likely to develop MDH [15]. Therefore, it is prudent to limit the number and dosage of medications to sick patients to the absolutely necessary, even antipyretics [12]. Suppressing early excessive T-cell stimulation with corticosteroids and having medication-free periods between adding drugs might reduce the risk. In our particular patient, the course was very convoluted with many unpredictable severe complications that led to her demise.

\section{Conflict of interest}

The authors declare that they have no conflict of interest. 


\section{References}

1. Szatkowski J, and Schwartz RA. (2015) Acute generalized exanthematous pustulosis (AGEP): A review and update. J Am Acad Dermatol; 73(5):843-848.

2. Kano Y, Inaoka M, and Shiohara T. (2004) Association between anticonvulsant hypersensitivity syndrome and human herpesvirus 6 reactivation and hypogammaglobulinemia. Arch Dermatol; 140(2):183-188.

3. Shiohara T, Inaoka M, and Kano Y. (2006) Drug-induced hypersensitivity syndrome (DIHS): a reaction induced by a complex interplay among herpesviruses and antiviral and antidrug immune responses. Allergol Int; 55(1):1-8.

4. Ushigome Y, Kano Y, Hirahara K, Shiohara T(2012) Human herpesvirus 6 reactivation in drug-induced hypersensitivity syndrome and DRESS validation score. Am J Med;125(7) e910.

5. Thomson AB, Sauve MD, Kassam N, Kamitakahara H (2010) Safety of the long-term use of proton pump inhibitors. World $\mathbf{J}$ Gastroenterol; 16(19):2323-2330.

6. Sandilands EA, and Bateman DN. (2009) Adverse reactions associated with acetylcysteine. Clin Toxicol (Phila); 47(2):8188.

7. Stella M, Clemente A, Bollero D, Risso D, Dalmasso P et al. (2007) Toxic epidermal necrolysis (TEN) and Stevens-Johnson syndrome (SJS): experience with high-dose intravenous immunoglobulins and topical conservative approach. A retrospective analysis. Burns; 33(4):452-459.
8. Bachot N, Revuz J, and Roujeau JC. (2003) Intravenous immunoglobulin treatment for Stevens-Johnson syndrome and toxic epidermal necrolysis: a prospective noncomparative study showing no benefit on mortality or progression. Arch Dermatol; 139(1):33-36.

9. Hung SI, Chung WH, Liu ZS, Chen CH, Hsih MS et al. (2010) Common risk allele in aromatic antiepileptic-drug induced Stevens-Johnson syndrome and toxic epidermal necrolysis in Han Chinese. Pharmacogenomics; 11(3):349-356.

10. Hu FY, Wu XT, An DM, Yan B, Stefan H et al. (2011) Pilot association study of oxcarbazepine-induced mild cutaneous adverse reactions with HLA-B*1502 allele in Chinese Han population. Seizure; 20(2):160-162.

11. Godhwani N, and Bahna SL. (2016) Antiepilepsy drugs and the immune system. Ann Allergy Asthma Immunol; 117(6):634-640.

12. Pichler WJ, Srinoulprasert Y, Yun J, Hausmann O. (2017) Multiple Drug Hypersensitivity. Int Arch Allergy Immunol; 172(3):129-138.

13. Khan DA. Treating patients with multiple drug allergies. (2013) Ann Allergy Asthma Immunol; 110(1):2-6.

14. Blumenthal KG, Saff RR, and Banerji A. (2014) Evaluation and management of a patient with multiple drug allergies. Allergy Asthma Proc; 35:197-203.

15. Picard D, Vellar M, Janela B, Roussel A, Joly P et al. (2015) Recurrence of drug-induced reactions in DRESS patients. J Eur Acad Dermatol Venereol; 29(4):801-804. 